\title{
THE RESTRICTION OF ADMISSIBLE MODULES TO PARABOLIC SUBALGEBRAS

\author{
BY
}

J. T. STAFFORD AND N. R. WALLACH ${ }^{1}$

\begin{abstract}
This paper studies algebraic versions of Casselman's subrepresentation theorem. Let $g$ be a semisimple Lie algebra over an algebraically closed field $F$ of characteristic zero and $\mathfrak{g}=\mathfrak{f} \oplus \mathfrak{a} \oplus \mathfrak{n}$ be an Iwasawa decomposition for $\mathfrak{g}$. Then $(\mathfrak{g}, \mathfrak{f})$ is said to satisfy property $(\mathfrak{n})$ if $\mathfrak{n} M \neq M$ for every admissible $(\mathfrak{g}, \mathfrak{f})$-module $M$. We prove that, if $(\mathfrak{g}, \mathfrak{f})$ satisfies property $(\mathfrak{n})$, then $\mathfrak{n} N \neq N$ whenever $N$ is a $(\mathfrak{g}, \mathfrak{f})$-module with $\operatorname{dim} N<$ card $F$. This is then used to show (purely algebraically) that $(\mathfrak{s l}(n, F), \mathfrak{s} o(n, F))$ satisfies property $(\mathfrak{n})$. The subrepresentation theorem for $\xi l(n)$ is an easy consequence of this.
\end{abstract}

1. Introduction. Let $F$ be a field of characteristic 0 . If $a$ is a Lie algebra over $F$ and if $\mathfrak{b} \subset \mathfrak{a}$ is a subalgebra then an $(\mathfrak{a}, \mathfrak{b})$-module is an $\mathfrak{a}$-module that splits into a direct sum of irreducible, finite-dimensional $\mathfrak{b}$-submodules. An $(\mathfrak{a}, \mathfrak{b})$-module $M$ is said to be admissible if $\operatorname{dim}_{\mathfrak{b}}(W, M)<\infty$ for each finite dimensional $\mathfrak{b}$-module $W . M$ is said to be finitely generated if it is finitely generated as an a-module.

Let $\mathfrak{g}_{0}$ be a semisimple Lie algebra over R. Let $\mathfrak{g}_{0}=\mathfrak{f}_{0} \oplus \mathfrak{a}_{0} \oplus \mathfrak{n}_{0}$ be an Iwasawa decomposition of $\mathfrak{g}_{0}$. Let $\mathfrak{g}, \mathfrak{f}, \mathfrak{a}$ and $\mathfrak{n}$ denote the respective complexifications of $\mathfrak{g}_{0}$, $\mathfrak{f}_{0}, \mathfrak{a}_{0}$ and $\mathfrak{n}_{0}$.

Let $G$ be a connected Lie group with Lie algebra $g_{0}$ and finite center. Let $K \subset G$ be the connected subgroup of $G$ corresponding to $\mathfrak{f}_{0}$. Then a $(g, K)$-module is a $(g, \mathfrak{f})$-module $M$ such that each of the irreducible $\mathrm{f}$-submodules of $M$ integrates to a representation of $K$. A $(g, K)$-module is said to be admissible if it is admissible as a $(g, \mathfrak{l})$-module.

A fundamental result of Casselman is

THEOREM 1. If $M$ is a nonzero admissible, finitely generated $(\mathrm{g}, K)$-module then $\mathfrak{n} M \neq M$.

Theorem 1 is equivalent to Casselman's strengthening of Harish-Chandra's subquotient theorem (cf. [5, 9]). Let us sketch the proof of this assertion. Let $M=\{m \in$ $\left.K|\operatorname{Ad}(m)|_{\mathfrak{a}}=I\right\}, A=\exp \mathfrak{a}_{0}, N=\exp \mathfrak{n}_{0}$. Let $\mathfrak{m}$ be the complexified Lie algebra of $M$; let $V$ be an admissible finitely generated $(\mathfrak{g}, K)$-module such that $V \neq \mathfrak{n} V$.

Received by the editors April 3, 1981.

1980 Mathematics Subject Classification. Primary 17B10, 17B20, 22 E47.

Key words and phrases. Semisimple Lie algebra, parabolic subalgebra, admissible (Harish-Chandra)module, subrepresentation theorem, Artin-Rees property.

'While this research was conducted, both authors were visiting Brandeis University. The first author was supported by a NATO Research Fellowship. The second was partially supported by an NSF grant. 
Then Theorem 5.2 implies that

$$
\operatorname{dim} V / \mathfrak{n} V<\infty
$$

Clearly, $V / \mathfrak{n} V$ is an ( $m \oplus \mathfrak{a} \oplus \mathfrak{n}, M$ )-module. Since $A N$ is simply connected $V / \mathfrak{n} V$ integrates to a $B=M A N$ representation. Let $(\sigma, H)$ be an irreducible quotient of $V / \mathfrak{n} V$ and let $q: V \rightarrow H$ be the $(\mathrm{m} \oplus a \oplus \mathfrak{n}, M)$-module projection. Define $X_{\infty}^{\sigma}$ to be the space of all $C^{\infty}$ maps $f$ mapping $G$ to $H$ such that $\mathfrak{f}(b g)=\sigma(b) \mathfrak{f}(g)$ for $b \in B$ and $g \in G$. If $x \in G$ define $\left(\pi_{\sigma}(x) f\right)(g)=f(g x)$. Let $X^{\sigma}$ denote the space of all $f \in X_{\infty}^{\sigma}$ such that $\pi_{\sigma}(K) f$ spans a finite dimensional space.

If $f \in X^{\sigma}$ and $x \in \mathfrak{g}_{0}$ define

$$
(x \cdot f)(g)=\left.\frac{d}{d t} f(g \exp t x)\right|_{t=0} .
$$

Then $X^{\sigma}$ is a $(g, K)$-module under this action.

Define $C(v)(n a k)=\sigma(n a) q(k \cdot v), n \in N, a \in A$ and $k \in K$. Then $C(v) \in X^{\sigma}$ and $C: V \rightarrow X^{\sigma}$ is a $(g, K)$-module homomorphism (this is Frobenius reciprocity). The $(g, K)$-modules $X^{\sigma}$ are elements of the so-called principal series. If $V$ is irreducible then $C$ is injective since it is nonzero. Thus an irreducible, admissible $(g, K)$-module is isomorphic with a subrepresentation of a principal series representation. This is the subrepresentation theorem. Thus Theorem 1 implies the subrepresentation theorem. The converse is also easily shown. Indeed, if $(\sigma, H)$ is an irreducible representation of $B$ and if $C: V \rightarrow X^{\sigma}$ is a $(\mathrm{g}, K)$-homomorphism, define $\delta_{C}(v)=C(v)(1)$. Then $\delta_{C}(\mathrm{n} V)=C(\mathrm{n} V)(1)=(\mathrm{n} C(V))(1)=0$ since $f(n)=f(1)$ if $n \in N$ and $f \in X^{\sigma}$. This implies that if $\mathfrak{n} V=V$ then $C(v)(1)=0$ for all $v \in V$. But then $0=C(k \cdot v)(1)=C(v)(k)$ for $v \in V, k \in K$. If $f \in X^{\sigma}$ then clearly $f=0$ if and only if $\left.f\right|_{K}=0$. This proves the converse.

Casselman's proof of Theorem 1 involves a study of the asymptotic expansion of matrix entries of admissible representations of Lie groups. Thus, although the statement of Theorem 1 is quite algebraic its proof dips fairly heavily into analysis. It is therefore natural to ask for an algebraic proof of Theorem 1. In order to do this we first express the content of Theorem 1 in a purely algebraic form (that is, over arbitrary fields and without reference to the Lie groups $G \supset K$ ).

Let $g$ be a semisimple Lie algebra over an algebraically closed field $F$, of characteristic 0 . Let $\theta: \mathfrak{g} \rightarrow \mathfrak{g}$ be an involutive automorphism and let $\mathfrak{f}=\{X \in \mathfrak{g} \mid \boldsymbol{\theta} X$ $=X\}$. Let $\mathfrak{g}=\mathfrak{i} \oplus \mathfrak{a} \oplus \mathfrak{n}$ be an Iwasawa decomposition of $\mathfrak{g}$ (that is $\mathfrak{a}=\{X \in \mathfrak{g} \mid$ $\theta X=-X\}$ and $[\mathfrak{a}, \mathfrak{a}]=0,[\mathfrak{a}, \mathfrak{n}] \subset \mathfrak{n}, \mathfrak{n}$ nilpotent and ad $\mathfrak{a}$ acts semisimply on $\mathfrak{g}$ ). We say that $(\mathfrak{g}, \mathfrak{f})$ has property $(\mathfrak{n})$ if, for each $M$, a nonzero, admissible, finitely generated $(\mathfrak{g}, \mathfrak{f})$-module $\mathfrak{n} M \neq M$ (i.e., $H_{0}(\mathfrak{n}, M) \neq(0)$ ).

If $F=\mathbf{C}$ and if it is shown that $(\mathfrak{g}, \mathfrak{f})$ has property $\mathfrak{n}$ for the pairs associated with semisimple Lie groups then Theorem 1 follows.

The purpose of this article is to give algebraic proofs of the property $(n)$ for certain classes of pairs $(\mathfrak{g}, \mathfrak{f})$ (we will describe them later in this introduction).

If $n$ is abelian then property $(\mathfrak{n})$ is an easy consequence of the Artin-Rees lemma of commutative algebra (see Proposition 3.1 and [2]). For nonabelian $\mathfrak{n}$ we use a generalization of that lemma due to McConnell [7]. We include a proof of this result 
(see Theorem 2.1) since we were able to give a slightly different result with a considerably easier proof.

In order to carry out our proofs of the property $(n)$ we prove the following result (see Theorem 5.10).

THEOREM 2. If $(\mathfrak{g}, \mathfrak{l})$ satisfies property $(\mathfrak{n})$ and if $M$ is a nonzero $(\mathfrak{g}, \mathfrak{f})$-module with $\operatorname{dim} M<\operatorname{card} F$ then $\mathfrak{n} M \neq M$.

The point of Theorem 2 is that admissibility and finite generation have been dropped.

We now give a more precise description of what we prove relative to property $(\mathfrak{n})$. Set $\mathfrak{m}=\{X \in \mathfrak{f} \mid[X, \mathfrak{a}]=0\}$. Put $\mathfrak{p}=\mathfrak{m} \oplus \mathfrak{a} \oplus \mathfrak{n}$.

TheOREM 3. If $\operatorname{dim} \mathfrak{a}=1$ and $M \neq(0)$ is $a(\mathfrak{p}, \mathfrak{m})$-module finitely generated as a $U(\mathfrak{n})$-module, then $\mathfrak{n} M \neq M$.

The case of Theorem 3 when $[n, n]=(0)$ is due to Casselman and Osborne [2].

Theorem 3 suggests that the property $(n)$ might be a property of $p$-modules. In $\S 4$ we give an example for $\$ l(3, F)$ which shows that Theorem 3 breaks down if $\operatorname{dim} a>1$.

The example in $\S 4$ actually shows that there exists $M$, an $\mathfrak{s} l(3, F)$-module, such that

(a) $M=U(\mathfrak{n}) \cdot v$,

(b) $\mathfrak{n} \cdot M=M$.

This example shows that Casselman's theorem is a theorem about $(\mathfrak{g}, \mathfrak{f})$-modules, rather than just about $(\mathfrak{p}, \mathfrak{m})$-modules.

Our next results involve the pairs $(\mathfrak{l} l(n, F), \mathfrak{b} o(n, F))$ where $a$ is the space of diagonal elements of $\mathfrak{g} l(n, F)$ and $\mathfrak{n}$ is the space of upper triangular matrices with zeros along the main diagonal. Set $\mathfrak{p}_{n-1}$ equal to the maximal parabolic subalgebra consisting of elements of $\mathfrak{g} l(n, F)$ of the form

$$
\left[\begin{array}{l|l}
A & b \\
\hline 0 & d
\end{array}\right]
$$

with $A$ an $n-1 \times n-1$ matrix. Then $\mathfrak{p}_{n-1} \cong g l(n-1, F) \oplus F^{n-1}$ (a semidirect product with $g l(n-1, F)$ acting on $F^{n-1}$ in the natural manner). We prove

THEOREM 4. If $M \neq 0$ is a $\left(\mathfrak{p}_{n}, \mathfrak{g} o(n, F)\right)$-module finitely generated under $U\left(\mathrm{n} \oplus F^{n}\right)$ then $\left(\mathfrak{n} \oplus F^{n}\right) M \neq M$.

This result easily implies property $(\mathfrak{n})$ for $(\xi l(n, F), \xi o(n, F))$. The proof of Theorem 4 proceeds by induction using Theorem 2 in a serious way. Theorem 4 suggests that property $(\mathfrak{n})$ is really a property of modules over maximal parabolic subalgebras. We note that the example of $\S 4$ shows that the condition that $M$ is a $\left(\mathfrak{p}_{n}, \mathfrak{S} o(n, F)\right)$-module cannot be dropped.

2. The Artin-Rees property and its immediate consequences. Let $R$ be a Noetherian ring (not necessarily commutative). Let $I \subset R$ be a two sided ideal. Then $I$ is said to have the $A-R$ property if given any finitely generated $R$-modules, $N \subset M$, then there 
exists an integer $k$ such that $I N \supset\left(I^{k} M\right) \cap N$. The Artin-Rees lemma asserts that any ideal of a commutative Noetherian ring has the A-R property. Let $R[t]$ be the set of polynomials in the commuting indeterminate $t$ with coefficients in $R$. Then $I$ is said to have the strong $A-R$ property if the ring

$$
R^{*}=R \oplus t I \oplus t^{2} I^{2} \oplus \ldots
$$

is Noetherian. The usual proofs of the Artin-Rees lemma show that the strong A-R property implies the A-R property (cf. Zariski and Samuel [10, p. 55], Atiyah and Macdonald [1, p. 107]).

Theorem 2.1. Let $\mathfrak{g}$ be a finite dimensional Lie algebra. Let $\mathfrak{n} \subset U(\mathfrak{g})$ be a finite dimensional Lie subalgebra such that

(1) $\mathfrak{n}$ is nilpotent as a Lie algebra,

(2) $[\mathrm{g}, \mathrm{n}] \subseteq \mathrm{n}$.

Then $I=\mathfrak{n} U(\mathrm{~g})$ has the strong $A-R$ property. Consequently $I$ has the $A-R$ property.

Proof. Define $\mathfrak{n}_{1}=\mathfrak{n}$ and $\mathfrak{n}_{i+1}=\left[\mathfrak{n}, \mathfrak{n}_{i}\right], i \geqslant 1$. Then there exists $d$ such that $\mathfrak{n}_{d} \neq 0$ and $\mathfrak{n}_{d+1}=(0)$. Set $\mathfrak{g}(t)=\mathfrak{g} \oplus t \mathfrak{n}_{1} \oplus t^{2} \mathfrak{n}_{2} \oplus \cdots \oplus t^{d} \mathfrak{n}_{d}$. Then $\mathrm{g}(t)$ is a finite dimensional Lie subalgebra of $U(\mathfrak{g})[t]$. Hence there is a canonical homomorphism $\phi: U(g(t)) \rightarrow U(g)[t]$. Clearly, $\phi(U(g(t)))=U(g)^{*}$. So $U(g)^{*}$ is Noetherian since $U(\mathfrak{g}(t))$ is Noetherian.

The above proof was inspired by an argument in Kostant [4].

The above result was only previously known when $g$ was solvable [7]. However if $g$ is nilpotent then any two sided ideal of $U(\mathfrak{g})$ has the A-R property [7]. This does not seem to follow from Theorem 2.1.

LEMMA 2.2. Let $R$ be a Noetherian ring. Let $I \subset R$ have the $A-R$ property. Suppose that $M$ is a finitely generated left $R$-module such that $M=I M$. If $N \subset M$ is $a$ submodule of $M$ then $I N=N$.

PROOF. There exists an integer $k$ such that

$$
I N \supseteq\left(I^{k} M\right) \cap N=M \cap N=N .
$$

So $I N=N$.

Corollary 2.3 (To Theorem 2.1). Let $\mathrm{g}$ be a finite dimensional Lie algebra over a field $F$. Let $u \in U(\mathfrak{g})$ be such that $[\mathfrak{g}, u] \subset F u$. If $M$ is a finitely generated $U(\mathfrak{g})$-module such that $u M=M$ then $u$ acts injectively on $M$.

Proof. Theorem 2.1 implies that $I=u U(g)=U(g) u$ has the A-R property. Let $m \in M$ and put $N=U(\mathfrak{g}) m$. Lemma 2.2 implies that $I N=N$. Hence there is $g \in U(g)$ such that $m=g u \cdot m$. Thus $u m=0$ implies that $m=0$.

3. Some analogues of Nakayama's lemma. In this section we apply the A-R property in the case when the Lie algebras contain abelian or "almost" abelian normal subalgebras. In particular we prove Theorem 3 of the introduction. Throughout this section $F$ denotes a field of characteristic 0 . 
Proposition 3.1. Let $\mathrm{g}$ be a finite dimensional Lie algebra over $F$ such that $\mathrm{g}=F H \oplus V$ where $V$ is a subspace of $\mathrm{g}$ and $H$ an element of $\mathrm{g}$ with

(i) $[V, V]=0$,

(ii) $[H, V] \subseteq V$ and ad $H / V$ diagonalizes with distinct eigenvalues $\lambda_{1}, \ldots, \lambda_{k}$ such that $\sum_{1}^{k} n_{i} \lambda_{i}=0$ with each $n_{i} \geqslant 0 \in \mathbf{Z}$ implies that $n_{i}=0$ for all $i$.

Let $M$ be a nonzero g-module, finitely generated as a $U(V)$-module. Then $V M \neq M$.

Proof. Suppose $V M=M$ and write $M=\sum_{1}^{k} U(V) m_{i}$ for some $m_{i} \in M$. Let $I=V U(V)$. Then by Corollary 2.2 there exist, for $1 \leqslant i \leqslant k, u_{i} \in I$ such that $\left(u_{i}-1\right) m_{i}=0$. Define $\omega \in I$ by $\Pi_{1}^{k}\left(u_{i}-1\right)=\omega-1$. Furthermore $(\omega-1) M=$ $\pm \Pi\left(u_{j}-1\right) \sum U(V) m_{i}= \pm \sum U(V) \Pi\left(u_{j}-1\right) m_{i}=0$. On $U(V)$, ad $H$ diagonalizes with eigenvalues $\sum_{1}^{k} n_{i} \lambda_{i}$ with $n_{i} \geqslant 0 \in \mathbf{Z}$. Thus by hypothesis (ii) we can write $\omega=\sum_{1}^{k} \omega_{i}$ where each $0 \neq \omega_{i} \in V U(V)$ and ad $H \omega_{i}=\mu_{i} \omega_{i}$ for distinct nonzero elements $\mu_{i} \in F$. Now write $X=\{x \in V U(V): x m=m$ for all $m \in M\}$. Then given $x \in X$ and $m \in M$,

$$
[H, x] m=H x m-x(H m)=H m-H m=0 .
$$

Thus $[H, x] M=0$. In particular $\omega \in X$. So set $v_{1}=\omega$ and for $i>1$ put $v_{i}=v_{i-1}$ $-\mu_{i-1}^{-1}\left[H, v_{i-1}\right]$. Then each $v_{i} \in X$ and, by induction, $v_{i}=\sum_{j=i}^{k} c_{j} \omega_{j}$ for some $c_{j}=c_{j}(i) \in F$. In particular $v_{k}=c \omega_{k} \in X$ for some $0 \neq c \in F$. Thus for any $m \in M, 0=\left[H, v_{k}\right] m=\mu_{k} v_{k} M=\mu_{k} M$ as $v_{k} \in X$. This is only possible if $M=0$, giving the required contradiction.

Notes. (1) This lemma is an analogue of Nakayama's lemma in the sense that $V U(V)$ is the unique ad $H$ invariant maximal ideal of $U(V)$.

(2) It would seem reasonable that there should be a generalization of Proposition 3.1 , the case when $V$ is nilpotent. However the obvious generalization to the case when $V$ is the three dimensional Heisenberg algebra is false, as will be shown in the next section.

(3) The above proposition implies one of the main results in Casselman and Osborne [2].

The second result of this section gives one case in which the result of Proposition 3.1 does hold for a nilpotent subalgebra $V$, and for which the proof is still fairly easy. We will first fix some notation. Let $g$ be a semisimple Lie algebra, $\mathfrak{p}$ a proper parabolic of $\mathfrak{g}$. Then we can write $\mathfrak{p}=\mathfrak{m} \oplus \mathfrak{a} \oplus \mathfrak{n}$ where $\mathfrak{m}, \mathfrak{a}$ and $\mathfrak{n}$ are subalgebras of $\mathfrak{p}$ with $\mathfrak{n}$ normal (as in the introduction).

We assume that $\operatorname{dim} a=1$ for the remainder of this section.

Proposition 3.2. Let $M$ be a $(\mathfrak{p}, \mathfrak{m})$-module, finitely generated as a $U(\mathfrak{n})$-module. If $\mathrm{n} M=M$ then $M=(0)$.

Proof. The method of proof is to find a second module over a subalgebra of $U(\mathfrak{p})$ to which we can apply the result of Proposition 3.1. We first note some standard results about $\mathfrak{p}$ (see for example [5]). We can write $\mathfrak{a}=F H$ with $[H, \mathfrak{m}]=0$. Further, ad $H \mid \mathfrak{n}$ has two eigenvalues 1 , 2. So write $\mathfrak{n}_{j}=\{X \in \mathfrak{n} \mid[H, X]=j X\}$ for $j=1$ or 2 . Note that $\left[\mathfrak{n}_{1}, \mathfrak{n}_{1}\right] \subset \mathfrak{n}_{2}$. Given $Y \in \mathfrak{m}$, let ad $Y$ also denote the canonical 
extension of ad $Y$ to $S(\mathfrak{n})$, the symmetric algebra of $\mathfrak{n}$. Set

$$
S(\mathfrak{n})^{\mathfrak{m}}=\{f \in S(\mathfrak{n}) \mid \operatorname{ad} Y \cdot f=0 \text { for } Y \in \mathfrak{m}\} .
$$

Then $S(\mathfrak{n})^{\mathfrak{m}}=F\left[q_{1}, q_{2}\right]$ where $q_{i} \in S\left(\mathfrak{n}_{i}\right) \mathfrak{n}_{i}$ for $i=1,2$ and $q_{1}, q_{2}$ are homogeneous of degrees $j_{1}$ and $j_{2}$ respectively.

We regard $q_{1}$ and $q_{2}$ as elements of $U(n)$ under the symmetrization map and we first show that $U(\mathfrak{n})^{m}=F\left[q_{1}, q_{2}\right]$. For, let $U^{k}(n)$ be the canonical filtration of $U(\mathfrak{n})$. Then, as an m-module under ad, $U^{k}(\mathfrak{n}) / U^{k-1}(\mathfrak{n})=S^{k}(\mathfrak{n})$, the homogeneous elements of $S(\mathfrak{n})$ of degree $k$. Let $J_{k}=\Sigma\left\{F q_{1}^{a} q_{2}^{b} \mid a j_{1}+b j_{2}=k\right\} \subset U(\mathfrak{n})$. Then $U^{k}(\mathfrak{n})^{\mathfrak{m}}=J_{k}+U^{k-1}(\mathfrak{n})^{\mathfrak{m}}$, as claimed.

Suppose $M \neq 0$ and $\mathfrak{n} M=M$. As an $m$-module $M=\bigoplus M_{\gamma}$ where $M_{\gamma}$ is a direct sum of irreducible finite dimensional $m$-modules each isomorphic with a fixed m-module, say $V_{\gamma}$. Now if $M \neq 0$ then $M_{\gamma} \neq 0$ for some $\gamma$. Let $V_{\gamma}^{*}$ be the m-module contragradient to $V_{\gamma}$. Extend $V_{\gamma}^{*}$ to a g-module by defining $H V_{\gamma}^{*}=\mathfrak{n} V_{\gamma}^{*}=0$. Then it is easily seen that:

(a) $M \otimes V_{\gamma}^{*}$ is finitely generated as a $U(\mathfrak{n})$-module,

(b) $\mathfrak{n}\left(M \otimes V_{\gamma}^{*}\right)=M \otimes V_{\gamma}^{*}$,

(c) $\left(M \otimes V_{\gamma}^{*}\right)^{\mathfrak{m}} \neq 0$.

Set $N=U(\mathfrak{n})\left(M \otimes V_{\gamma}^{*}\right)^{\mathfrak{m}}$. Then by Lemma $2.2, \mathfrak{n} N=N$. Furthermore, $N$ is a $\mathrm{g}$-module since $\left(M \otimes V_{\gamma}^{*}\right)^{\mathfrak{m}}$ is $\mathfrak{m} \oplus \mathfrak{a}$ invariant. By (a), $M \otimes V_{\gamma}^{*}$, and hence $N$, is finitely generated as a $U(\mathfrak{n})$-module. So choose $m_{1}, \ldots, m_{k} \in\left(M \otimes V_{\gamma}^{*}\right)^{\mathfrak{m}}$ such that $N=\Sigma U(\mathfrak{n}) m_{i}$.

Since $m$ acts semisimply on $U(\mathfrak{n})$, there exists a projection of $U(\mathfrak{n})$ onto $U(\mathfrak{n})^{\mathfrak{m}}$. Given $u \in U(\mathfrak{n})$ let $u^{0}$ denote the image of $u$ under this projection. Now if $n \in N^{\mathfrak{m}}$ then $n=\sum a_{i} m_{i}$ for some $a_{i} \in U(\mathfrak{n})$ and so $n=\sum a_{i}^{0} m_{i}$. Thus $N^{\mathfrak{m}}$ is a finitely generated $U(\mathfrak{n})^{\mathfrak{m}}=F\left[q_{1}, q_{2}\right]$-module.

Set $\mathfrak{g}=F H \oplus F q_{1} \oplus F q_{2}$. Then $\left[H, q_{1}\right]=j_{1} q_{1},\left[H, q_{2}\right]=j_{2} q_{2}$ and $\left[q_{1}, q_{2}\right]=0$. So $\mathfrak{g}$ satisfies the hypotheses of Proposition 3.1 and $N^{\mathfrak{m}}$ is an $\mathfrak{g}$-module.

Further, given $n \in N^{\mathfrak{m}}$ the A-R property implies that there exists $u \in \mathfrak{n} U(\mathfrak{n})$ such that $u n=n$ and hence $u^{0} n=n$. But $u^{0} \in q_{1} F\left[q_{1}, q_{2}\right]+q_{2} F\left[q_{1}, q_{2}\right]$ whence $N^{\mathfrak{m}}=$ $q_{1} N^{\mathfrak{m}}+q_{2} N^{\mathfrak{m}}$. Now Proposition 3.1 with $\mathfrak{g}=\mathfrak{g}, V=F q_{1} \oplus F q_{2}$ implies that $N^{\mathfrak{m}}=$ 0 . This contradiction proves the proposition.

4. A family of $\mathfrak{s} l(3, F)$-modules. In this section we study a family of modules for $\mathfrak{g} l(3, F)$ whose existence implies, in particular, that Proposition 3.1 is false if $V$ is replaced by the Heisenberg algebra.

Let $E_{i j} \in \operatorname{End}\left(F^{3}\right)$ be defined by $E_{i j} e_{k}=\delta_{j k} e_{i}$ (where $e_{1}, e_{2}, e_{3}$ is the standard basis of $F^{3}$ ). We set $x=E_{12}, y=E_{23}, z=E_{13}, h=E_{11}-E_{33}, h^{\prime}=E_{11}-E_{22}$, $\bar{x}=E_{21}, \bar{y}=E_{32}, \bar{z}=E_{31}$. Set $\mathfrak{n}=F x+F y+F z$. Let $M=\bigoplus F v_{k, l}$, the sum over $k \in \mathbf{Z}, l \in \mathbf{Z}$, with $k \geqslant 0$. Set $v_{k, l}=0, k<0$. We define the following linear maps for $\alpha \in F$ :

$$
\begin{aligned}
& X_{\alpha} v_{k, l}=v_{k+1, l}, \quad Y_{\alpha} v_{k, l}=v_{k, l+1}-k v_{k-1, l-1}, \quad Z_{\alpha} v_{k, l}=v_{k, l-1}, \\
& H_{\alpha} v_{k, l}=3 v_{k+1, l+2}+(k-2 l) v_{k, l}, \quad H_{\alpha}^{\prime} v_{k, l}=(2 k-l+\alpha) v_{k, l} \\
& \bar{X}_{\alpha} v_{k, l}=v_{k+1, l+4}+k(1-k-\alpha+1) v_{k-1, l}+(\alpha-2-l) v_{k, l+2}, \\
& \bar{Y}_{\alpha} v_{k, l}=-2 v_{k+2, l+3}+(\alpha+l) v_{k+1, l+1}, \quad \bar{Z}_{\alpha}=-\left[\bar{X}_{\alpha}, \bar{Y}_{\alpha}\right] .
\end{aligned}
$$


Lemma 4.1. Suppose that $A: M \rightarrow M$ is a linear map that commutes with $X_{\alpha}, Y_{\alpha}, Z_{\alpha}$ and $H_{\alpha}^{\prime}$. Then $A=\lambda I$ for some $\lambda \in F$.

Proof. Set $A v_{k, l}=\sum a_{r, s ; k, l} v_{r, s}$. Then $\left[A, H_{\alpha}^{\prime}\right]=0$ implies

$$
a_{r, s ; k, l}=0 \text { if } 2 r-s \neq 2 k-l \text {. }
$$

$\left[A, X_{\alpha}\right]=0$ implies

$$
a_{r+1, s ; k+1, l}=a_{r, s ; k, l}
$$

$\left[A, Z_{\alpha}\right]=0$ implies

$$
a_{r, s+1 ; k, l+1}=a_{r, s ; k, l} .
$$

(2), (3) combined with $\left[A, Y_{\alpha}\right]=0$ imply

$$
a_{r, s ; k, l}=0 \text { if } r \neq k \text {. }
$$

(1) now implies that $A v_{k, l}=a_{k, l} v_{k, l}$.

Now (1), (2) clearly imply the lemma.

TheOREM 4.2. The correspondence $x \rightarrow X_{\alpha}, y \rightarrow Y_{\alpha}, z \rightarrow Z_{\alpha}, h \rightarrow H_{\alpha}, h^{\prime} \rightarrow H_{\alpha}^{\prime}$, $\bar{x} \rightarrow \bar{X}_{\alpha}, \bar{y} \rightarrow \bar{Y}_{\alpha}$ and $\bar{z} \rightarrow \bar{Z}_{\alpha}$ defines an $\$ l(3, F)$-module structure on $M$ which we denote by $M_{\alpha}$.

$M_{\alpha}$ has the following properties:

(1) $U(\mathfrak{n}) v_{0,0}=M_{\alpha}$.

(2) $z M_{\alpha}=M_{\alpha}$.

Proof. Assuming that $M_{\alpha}$ is indeed an $\mathfrak{s} l(3, F)$-module, properties (1) and (2) are clear. Indeed, $M_{\alpha}=\Sigma F x^{k} y^{l} v_{0,0}+\sum F x^{k} z^{l} v_{0,0}$ and $v_{k, l}=z v_{k, l+1}$.

To prove the theorem we must only demonstrate that the commutation relations come out correctly. This is straightforward (but tedious). The only possible difficulty is to show that $\left[\bar{Z}_{\alpha}, \bar{X}_{\alpha}\right]=\left[\bar{Z}_{\alpha}, \bar{Y}_{\alpha}\right]=0$. However, Lemma 4.1 implies (assuming that the reader has verified all of the other commutation relations) that $\left[\bar{Z}_{\alpha}, \bar{X}_{\alpha}\right]=\mu I$ and $\left[\bar{Z}_{\alpha}, \bar{Y}_{\alpha}\right]=\lambda I$ with $\lambda, \mu \in F$. Also $\left[H_{\alpha}^{\prime},\left[\bar{Z}_{\alpha}, \bar{X}_{\alpha}\right]\right]=-3\left[\bar{Z}_{\alpha}, \bar{X}_{\alpha}\right]$; hence $0=-3 \mu I$. Thus $\mu=0$. Similarly, $\left[H_{\alpha},\left[\bar{Z}_{\alpha}, \bar{Y}_{\alpha}\right]\right]=-3\left[\bar{Z}_{\alpha}, \bar{Y}_{\alpha}\right]$ and hence $-3 \lambda I=0$.

We note that in $\$ 6$ we will prove a result (Theorem 3 in the introduction) that has the following corollary. Suppose that $V$ is an $\mathfrak{g} l(3, F)$-module such that:

(a) $x-\bar{x}$ has a nonzero eigenvector,

(b) $V$ is finitely generated under $U(\mathfrak{n})$.

Then $V \neq n V$.

5. The property $(\mathfrak{n})$. The goal of this section is to prove Theorem 2 of the introduction. Throughout this section $g$ will be a semisimple Lie algebra over an algebraically closed field $F$ of characteristic 0 . Let $\theta$ be a nontrivial involutive automorphism of $g$. We set

$$
\mathfrak{l}=\{X \in \mathfrak{g} \mid \boldsymbol{\theta} X=X\}, \quad V=\{X \in \mathfrak{g} \mid \boldsymbol{\theta} X=-X\} .
$$

We fix $a \subset V$ a subspace satisfying

(1) $[a, a]=0$.

(2) If $H \in \mathfrak{a}$ then ad $H$ is a semisimple endomorphism of $g$.

(3) $\{X \in \mathfrak{g} \mid[X, \mathfrak{a}]=0\} \cap V=\mathfrak{a}$.

Such an a $\subset V$ always exists (cf. Dixmier [3, p. 58, 1.13.6]). 
If $\lambda \in \mathfrak{a}^{*}$ the set $\mathrm{g}^{\lambda}=\{X \in \mathfrak{g} \mid$ ad $H \cdot X=\lambda(H) X, H \in \mathfrak{a}\}$. Clearly $\mathfrak{g}=\bigoplus_{\lambda} \mathfrak{g}^{\lambda}$. Set $\Lambda=\left\{\lambda \in a^{*} \mid \lambda \neq 0, g^{\lambda} \neq 0\right\}$. Fix $\Lambda^{+} \subset \Lambda$ satisfying

(a) $\Lambda^{+} \cup-\Lambda^{+}=\Lambda$.

(b) $\Lambda^{+} \cap-\Lambda^{+}=\varnothing$.

(c) If $\mu, \lambda \in \Lambda^{+}$and $\lambda+\mu \in \Lambda$ then $\lambda+\mu \in \Lambda^{+}$.

Such a $\Lambda^{+}$exists (cf. [3, p. 54]); fix it. We set $\mathfrak{n}=\Sigma_{\lambda \in \Lambda^{+}} \mathfrak{g}^{\lambda}$ and $m=g^{0} \cap \mathfrak{f}$. Putting $\overline{\mathfrak{n}}=\theta \mathfrak{n}$, we have

(4) $\mathfrak{g}=\mathfrak{f} \oplus \mathfrak{a} \oplus \mathfrak{n}=\overline{\mathfrak{n}} \oplus \mathfrak{m} \oplus \mathfrak{a} \oplus \mathfrak{n}$.

Let $\mathfrak{h} \subset \mathfrak{g}$ be a Cartan subalgebra of $\mathfrak{g}$ such that $\mathfrak{h} \cap V=\mathfrak{a}$ and $\theta \mathfrak{h}=\mathfrak{h}$ (see $[3, \mathrm{p}$. $58,1.13 .7])$. Let $\Delta$ be the root system of $(\mathfrak{g}, \mathfrak{h})$. Let for $\alpha \in \Delta, \mathfrak{g}_{\alpha}=\{X \in \mathfrak{g} \mid$ ad $H$. $X=\alpha(H) X, H \in \mathfrak{h}\}$. Fix a system $\Delta^{+}$of positive roots for $\Delta$ such that, if $\mathfrak{n}^{+}=$ $\Sigma_{\alpha \in \Delta^{+}} \mathfrak{g}_{\alpha}$ then $\mathfrak{n} \subset \mathfrak{n}^{+}$.

Let $M$ be a $(\mathfrak{g}, \mathfrak{f})$-module. Then $M$ is said to be admissible if, for each finite dimensional $\mathrm{f}$-module $W$,

$$
\operatorname{dim} \operatorname{Hom}_{\mathfrak{t}}(W, M)<\infty .
$$

Definition 5.1. $(\mathfrak{g}, \mathfrak{l})$ is said to have property $(\mathfrak{n})$ if whenever $M$ is an admissible $(\mathrm{g}, \mathfrak{f})$-module, $\mathfrak{n} M=M$ implies $M=(0)$.

Before we get to the main results of this section we recall two theorems and we develop some formalism.

THEOREM 5.2 (OSBORNE, CF. [8]). If $M$ is an admissible $(\mathfrak{g}, \mathfrak{l})$-module that is finitely generated as a $U(\mathfrak{g})$-module then $M$ is finitely generated as a $U(\mathfrak{n})$-module.

TheOrem 5.3 (HARish-ChANDRA, CF. [9]). Let $M$ be $a(\mathfrak{g}, \mathfrak{l})$-module finitely generated as a $U(\mathfrak{g})$-module. Let $Z(g)$ be the center of $U(\mathfrak{g})$. If, for each $m \in M$, $\operatorname{dim} Z(g) \cdot m<\infty$ then $M$ is admissible.

COROllary 5.4. If $M$ is an irreducible $(\mathfrak{g}, \mathfrak{f})$-module then $M$ is admissible.

Proof. Quillen's lemma, cf. Dixmier [3, p. 88, 2.6.6], implies that there exists $\chi$ : $Z \rightarrow F$ such that $z \cdot m=\chi(z) m$ for $m \in M, z \in Z(g)$. Now apply 5.3.

The following lemma is due originally to J. Dixmier (see [3, Note 2.8.9, p. 97]). We include a proof for the reader's convenience.

LEMMA 5.5. Let $V$ be a vector space of dimension strictly less than the cardinality of $F$. If $T$ is an endomorphism of $V$ then there exists $\lambda \in F$ such that $T-\lambda$ is not bijective.

Proof. Let $F(x)$ be the field of rational functions in an indeterminate $x$. Then

$$
\operatorname{dim}_{F} F(x) \geqslant \text { cardinality }(F) \text {. }
$$

Indeed, it is easily seen that the elements $\left\{(x-\lambda)^{-1} \mid \lambda \in F\right\}$ are linearly independent over $F$.

Suppose $T-\lambda$ is bijective for all $\lambda \in F$. Fix $v \in V, v \neq 0$, and define $\phi$ : $F(x) \rightarrow V$ by

$$
\phi\left(\prod_{i=1}^{r}\left(x-\lambda_{i}\right)^{-n_{i}} \cdot p(x)\right)=\prod_{i=1}^{r}\left(T-\lambda_{i}\right)^{-n_{i}} \cdot p(T) v
$$


for $\lambda_{1}, \ldots, \lambda_{r} \in F$ and $p \in F[X]$. Suppose that $\phi(p / q)=0$. Then $p(T) v=0$. This implies that $T-\mu$ is not injective for some root $\mu$ of $p$. Thus $\phi$ is injective. Since $\operatorname{dim} V<\operatorname{card}(F)$ this combined with (1) is a contradiction.

If $M$ is a $g$-module, set $M^{*}[\mathfrak{n}]=\left\{\mu \in M^{*} \mid \mathfrak{n}^{k} \cdot \mu=0\right.$ for some $k$ (depending on $\mu)\}$. Since $\operatorname{ad}(\mathfrak{n})$ acts nilpotently on $\mathfrak{g}, M^{*}[\mathfrak{n}]$ is a $\mathfrak{g}$-submodule of $M^{*}$. We note that $M \leadsto M^{*}[\mathrm{n}]$ is a functor from the category of $\mathfrak{g}$-modules to the category of $\mathrm{g}$-modules. For if $A: M \rightarrow M_{1}$ is a $\mathrm{g}$-module homomorphism then $A^{*}\left(M_{1}^{*}[\mathfrak{n}]\right) \subset$ $M^{*}[\mathrm{n}]$.

THEOREM 5.6. (i) If $M$ is a $\mathrm{g}$-module finitely generated as a $U(\mathfrak{n})$-module then $M^{*}[\mathrm{n}]$ is finitely generated as a $U(\mathfrak{g})$-module.

(ii) Let $M$ be as in (i). If $\bar{m} \in M / \cap_{k=1}^{\infty} \mathfrak{n}^{k} M$, then $\operatorname{dim} Z(\mathfrak{g}) \cdot \bar{m}<\infty$.

(iii) Let $M, N, P$ be g-modules that are finitely generated as $U(\mathfrak{n})$-modules. Let $0 \rightarrow M \rightarrow^{\alpha} N \rightarrow^{\beta} P \rightarrow 0$ be an $\mathrm{g}$-module exact sequence. Then

$$
0 \rightarrow P^{*}[\mathfrak{n}] \stackrel{\beta^{*}}{\rightarrow} N^{*}[\mathfrak{n}] \stackrel{\alpha^{*}}{\rightarrow} M^{*}[\mathfrak{n}] \rightarrow 0
$$

is an exact sequence.

Proof. We first derive some consequences of the assumption that $M$ is finitely generated as a $U(\mathfrak{n})$-module that will also be useful later. The most obvious consequence is $\operatorname{dim} M / \mathfrak{n}^{k} M<\infty$. Clearly, $M^{*}[\mathfrak{n}]=\cup_{k=1}^{\infty}\left(M / \mathfrak{n}^{k} M\right)^{*}$.

If $V$ is an a-module and if $\mu \in a^{*}$. set $V_{\mu}=\left\{v \in V \mid(H-\mu(H))^{k} v=0\right.$ for some $k$ and all $H \in \mathfrak{a}\}$. $\mathfrak{a}$ is abelian and $\left(M / \mathfrak{n}^{k} M\right)^{*}$ is finite dimensional; hence $\left(M / \mathfrak{n}^{k} M\right)^{*}=\bigoplus_{\mu}\left(M / \mathfrak{n}^{k} M\right)_{\mu}^{*}$. But then $M^{*}[\mathfrak{n}]=\bigoplus_{\mu} M^{*}[\mathfrak{n}]_{\mu}$.

Set $S=\left\{\mu \in \mathfrak{a}^{*} \mid(M / \mathfrak{n} M)_{\mu}^{*} \neq 0\right\}$; clearly $S$ is finite. We first prove:

(1) If $\mu \in \mathfrak{a}^{*}$ and $\left(M / \mathfrak{n}^{k+1} M\right)_{\mu}^{*} \neq 0$ then $\mu=\xi-\left(\lambda_{1}+\cdots+\lambda_{j}\right)$, with $\xi \in S$, $\lambda_{i} \in \Lambda^{+}$and $0 \leqslant j \leqslant k$. If there exists $v \in\left(M / \mathfrak{n}^{k+1} M\right)_{\mu}^{*}$ and $v \notin\left(M / \mathfrak{n}^{k} M\right)_{\mu}^{*}$ then $\mu=\xi-\lambda_{1}-\cdots-\lambda_{k}$ with $\lambda_{i} \in \Lambda^{+}$.

Indeed, if $v \in\left(M / \mathfrak{n}^{k+1} M\right)_{\mu}^{*}$ but $v \notin\left(M / \mathfrak{n}^{k} M\right)_{\mu}^{*}$, then there are elements $X_{j} \in$ $\mathfrak{g}_{\lambda_{i}}, \lambda_{i} \in \Lambda^{+}, i=1, \ldots, k$, so that $X_{1} \cdots X_{k} \cdot v \neq 0$ and $\mathfrak{n} \cdot X_{1} \cdots X_{k} \cdot v=0$. But

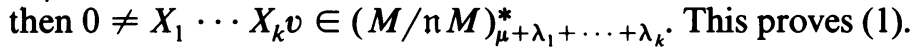

Let $H_{0} \in$ a be such that $\lambda\left(H_{0}\right) \geqslant 1$ for $\lambda \in \Lambda^{+}$. (Such an $H_{0}$ exists since we can take $H_{0}$ to be the element that satisfies $\lambda\left(H_{0}\right)=1$ for $\lambda \in \Lambda^{+}$a simple root.)

(2) If $\mu \in \mathfrak{a}^{*}$ and if $M^{*}[\mathfrak{n}]_{\mu} \neq 0$ then there exists $k$ so that

$$
M^{*}[\mathfrak{n}]_{\mu}=\left(M / \mathfrak{n}^{k+1} M\right)_{\mu}^{*} .
$$

If not then there would be an infinite sequence $k_{1}<k_{2}<\cdots<k_{n}<\cdots$ so that $\left(M / \mathfrak{n}^{k_{j}+1} M\right)_{\mu}^{*} \neq\left(M / \mathfrak{n}^{k_{j+1}+1} M\right)_{\mu}^{*}$. (1) would then imply $\mu=\xi_{i}-\lambda_{i, 1}-\cdots-\lambda_{i, k_{j}}$ with $\xi_{i} \in S$ and $\lambda_{i, j} \in \Lambda^{+}, i=1,2, \ldots$

But $S$ is a finite set. Hence by replacing $\left\{k_{i}\right\}$ by a subsequence we may assume $\xi_{i}=\xi, i=1,2, \ldots$ But then $(\xi-\mu)\left(H_{0}\right)=\lambda_{i, 1}\left(H_{0}\right)+\cdots+\lambda_{i, k_{i}}\left(H_{0}\right) \geqslant k_{i}, i=$ $1,2, \ldots$ This is ridiculous.

(2) immediately implies that

$$
\operatorname{dim} M^{*}[\mathfrak{n}]_{\mu}<\infty
$$


Let $Z$ denote the center of $U(g)$. If $V$ is a $Z$-module and if $\chi: Z \rightarrow F$ is a homomorphism then set

$$
V^{x}=\left\{v \in V \mid(z-\chi(z))^{k} v=0 \text { for some } k \text { and all } z \in Z\right\} .
$$

Clearly, $Z \cdot M^{*}[\mathfrak{n}]_{\mu} \subset M^{*}[\mathfrak{n}]_{\mu}$. Hence $M^{*}[\mathfrak{n}]_{\mu}=\bigoplus_{\chi} M^{*}[\mathfrak{n}]_{\mu}^{\chi}$ since $M^{*}[\mathfrak{n}]_{\mu}$ is finite dimensional. But then $M^{*}[\mathrm{n}]=\bigoplus_{\chi} M^{*}[\mathrm{n}]^{\chi}$.

(4) There exist $\chi_{1}, \ldots, \chi_{r}, r<\infty$, so that $M^{*}[n]^{\chi_{i}} \neq(0)(1 \leqslant j \leqslant r)$ and $M^{*}[n]=$ $\bigoplus_{i=1}^{r} M^{*}[\mathrm{n}]^{x_{i}}$

We note that $(M / \mathfrak{n} M)^{*}=\left\{v \in M^{*}[\mathfrak{n}] \mid \mathfrak{n} \cdot v=0\right\}$. Thus $Z \cdot(M / \mathfrak{n} M)^{*} \subset$ $(M / \mathfrak{n} M)^{*}$. But then $(M / \mathfrak{n} M)^{*}=\bigoplus_{i=1}^{r}\left((M / \mathfrak{n} M)^{*}\right)^{\chi_{i}}$ with $r<\infty$. If $v \in M^{*}[\mathrm{n}]^{x}$ then $v \in\left(\left(M^{*} / \mathfrak{n}^{k+1} M\right)^{*}\right)^{\chi}$ for some $\chi$. There exists $n \in U(\mathfrak{n})$ so that $0 \neq n \cdot v \in$ $\left((M / \mathfrak{n} M)^{*}\right)^{\chi}$. Hence $\chi=\chi_{i}$ for some $1 \leqslant i \leqslant r$. This proves (4).

Let $V=\left\{\Lambda \in \mathfrak{h}^{*} \mid\right.$ the Verma module with highest weight $\Lambda$ has infinitesimal character $\chi_{i}$ for some $1 \leqslant i \leqslant r$ \}. See Dixmier [3, Chapter 7] for the pertinent facts on Verma modules. In particular, $V$ is a finite set.

We are now ready to prove the theorem. We note that $m \cdot\left(M / n^{k} M\right)^{*} \subset$ $\left(M / \mathfrak{n}^{k} M\right)^{*}$. Hence the actin of $\mathfrak{n}^{+}$on $M^{*}[\mathfrak{n}]$ is locally nilpotent. This implies that if $\bar{M}$ is a nonzero subquotient of $M^{*}[\mathfrak{n}]$ then there exists $0 \neq v_{0} \in \bar{M}$ and $\mu \in \mathfrak{h} *$ such that $\mathfrak{n}^{+} \cdot v_{0}=0$ and $h \cdot v_{0}=\mu(h) v_{0}, h \in \mathfrak{h}$. But then $\mu \in V$ by the definition of $V$.

Put $V_{0}=\left\{\left.\Lambda\right|_{\mathfrak{a}} \mid \Lambda \in V\right\}$. Then $V_{0}$ is a finite set. Put $W=\bigoplus_{\mu \in V_{0}} M^{*}[\mathrm{n}]_{\mu}$. Then $\operatorname{dim} W<\infty$ by (3). Put $N=U(\mathfrak{g}) \cdot W$. If $\mu \in V_{0}$ then $\left(M^{*}[\mathrm{n}] / N\right)_{\mu}=0$. The above observation implies that $M^{*}[\mathrm{n}] / N=0$. Hence $N=M^{*}[\mathrm{n}]$. This proves (i).

To prove (ii) we note that our proof of (i) implies that there exist $v_{1}, \ldots, v_{s} \in$ $M^{*}[n]$, positive integers $k_{i}$ and homomorphisms $\chi_{i}$ of $Z$ to $F$ such that

(a) $M^{*}[\mathrm{n}]=\sum_{i=1}^{s} U(\mathrm{~g}) v_{i}$,

(b) $\left(z-\chi_{1}(z)\right)^{k_{i}} v_{i}=0, i=1, \ldots, s$ and $z \in Z$.

But then $\prod_{i=1}^{s}\left(z-\chi_{i}(z)\right)^{k_{i}} v=0, v \in M^{*}[\mathrm{n}]$. Hence if $m \in M$ then

$$
v\left(\prod_{i=1}^{s}\left({ }^{t} z-\chi_{i}(z)\right)^{k_{i}} \cdot m\right)=0, \quad v \in M^{*}[\mathrm{n}], z \in Z .
$$

Hence $\Pi_{i=1}^{s}\left({ }^{t} z-\chi_{i}(z)\right)^{k_{i}} M \subset \bigcap_{n=1}^{\infty} \mathfrak{n}^{n} M$. This implies (ii).

The only nontrivial part of (iii) is the surjectivity. We may assume that $M$ is a $\mathrm{g}$-submodule of $N$. We must show that $\left.N^{*}[\mathrm{n}]\right|_{M}=M^{*}[\mathrm{n}]$. Theorem 2.1 implies that for each $k$ a positive integer there is a positive integer $r(k)$ such that

$$
\mathfrak{n}^{r(k)} N \cap M \subset \mathfrak{n}^{k} M .
$$

Hence $\left(M / \mathfrak{n}^{k} M\right)^{*} \subset\left(M /\left(\mathfrak{n}^{r(k)} N\right) \cap M\right)^{*}$. Now

$$
M /\left(\mathfrak{n}^{r(k)} N\right) \cap M \subset N / \mathfrak{n}^{r(k)} N .
$$

Thus if $\lambda \in\left(M / \mathfrak{n}^{k} M\right)^{*}$ then $\lambda \in\left(M /\left(\mathfrak{n}^{r(k)} N\right) \cap M\right)^{*}$. Hence $\lambda$ extends to an element of $\left(N / \mathfrak{n}^{r(k)} N\right)^{*}$.

Part (iii) of the above theorem is part of a theory discovered independently by the second named author and $\mathrm{W}$. Casselman. A complete theory of the modules $M^{*}[\mathfrak{n}]$ will be developed in a forthcoming paper of Casselman and Wallach. 
THEOREM 5.7. Let $M$ be an admissible finitely generated $(g, \mathfrak{l})$-module. Suppose that $(\mathfrak{g}, \mathfrak{f})$ has property $(\mathfrak{n})$. Then $M$ has finite length.

Proof. The assumption that $(\mathfrak{g}, \mathfrak{l})$ has property $(\mathfrak{n})$ implies that if $V$ is a nonzero finitely generated $(g, \mathfrak{f})$-module then $V^{*}[\mathfrak{n}] \neq(0)$.

Theorem 5.6(i) says that $M^{*}[\mathfrak{n}]$ is finitely generated as a $U(\mathfrak{g})$-module. Hence $M^{*}[\mathfrak{n}]$ satisfies the ascending chain condition. Theorem 5.6(iii) implies that a descending chain

$$
M=M_{1} \supsetneqq M_{2} \supsetneqq \cdots \supsetneqq M_{k} \supsetneqq \cdots
$$

gives rise to an ascending chain

$$
\left(M / M_{1}\right)^{*}[\mathfrak{n}] \subsetneq\left(M / M_{2}\right) *[\mathfrak{n}] \subsetneq \cdots \subsetneq\left(M / M_{k}\right)^{*}[\mathfrak{n}] \subsetneq \cdots
$$

in $M^{*}[\mathrm{n}]$. The result follows.

Note. Theorem 5.7 is well known in the case $F=\mathrm{C}$ and $M$ is a $(g, K)$-module with $K$ compact. It is usually proven using the deep theorem of Harish-Chandra which states that the characters of admissible, irreducible, representations of semisimple Lie groups with finite center are locally $L^{1}$-functions.

If $M$ is an admissible finitely generated $(\mathfrak{g}, \mathfrak{f})$-module, set $E(M)=\left\{\mu \in \mathfrak{a}^{*} \mid\right.$ $\left.(M / \mathfrak{n} M)_{\mu}^{*} \neq 0\right\}$.

LEMMA 5.8. Let $M$ be an admissible finitely generated $(g, \mathfrak{l})$-module. Suppose that $M=M_{1} \supset M_{2} \supset \cdots \supset M_{d} \supset M_{d+1}=(0)$ is a composition series for $M$ such that $M_{i} / M_{i+1}=N_{i}$ is irreducible. (It exists whenever $(\mathfrak{g}, \mathfrak{l})$ satisfies property $(\mathfrak{n})$ by Theorem 5.7.) If $M^{*}[\mathfrak{n}]_{\mu} \neq(0)$ then $\mu=\xi-Q$ with $\xi \in \cup_{i=1}^{d} E\left(N_{i}\right)$ and $Q$ is a sum of elements of $\Lambda^{+}$.

Proof. By induction on $d$. If $d=1$ then the result is just (1) in the proof of 5.6. Suppose the result is true for $d$. Then we have the short exact sequence $0 \rightarrow M_{d} \rightarrow M$ $\rightarrow M / M_{d} \rightarrow 0$ which according to 5.6(iii) induces the short exact sequence

$$
0 \rightarrow\left(M / M_{d}\right) *[\mathrm{n}] \rightarrow M^{*}[\mathrm{n}] \rightarrow M_{d}^{*}[\mathrm{n}] \rightarrow 0 .
$$

Thus given a weight of $M^{*}[\mathfrak{n}]$ it must be a weight of $\left(M / M_{d}\right)^{*}[\mathfrak{n}]$ or a weight of $M_{d}^{*}[\mathrm{n}]$. The induction hypothesis now implies the result for $M$.

Let $z_{1}, \ldots, z_{l}$ be generators for $Z=Z(\mathfrak{g})$. Let $\hat{\mathfrak{f}}$ denote the set of isomorphism classes of irreducible finite dimensional $\mathfrak{t}$-modules. If $\gamma \in \hat{\mathfrak{t}}$ fix $V_{\gamma} \in \gamma$. If $\gamma \in \hat{\mathfrak{t}}$ and if $\chi: Z \rightarrow F$ is a homomorphism then put

$$
M_{\gamma, \chi, k}=U(\mathfrak{g}) \otimes_{U(\mathfrak{t})} V_{\gamma} / \sum_{i=1}^{l}\left(z_{i}-\chi\left(z_{i}\right)\right)^{k}\left(U(\mathfrak{g}) \otimes_{U(\mathfrak{i})} V_{\gamma}\right) .
$$

Theorem 5.3 implies that $M_{\gamma, \chi, k}$ is an admissible, finitely generated $(g, \mathfrak{f})$-module. We note that the inclusion

$$
\sum_{i=1}^{l}\left(z_{i}-\chi\left(z_{i}\right)\right)^{k+1}\left(U(\mathfrak{g}) \otimes_{U(\mathfrak{f})} V_{\gamma}\right) \subset \sum_{i=1}^{l}\left(z_{i}-\chi\left(z_{i}\right)^{k} U(\mathfrak{g}) \otimes_{U(\mathfrak{f})} V_{\gamma}\right)
$$


induces a surjective $\mathrm{g}$-module homomorphism $M_{\gamma, \chi, k+1} \stackrel{\psi_{k+1}}{\rightarrow} M_{\gamma, \chi, k}$ with $\operatorname{ker} \psi_{k+1}=\sum_{i=1}^{l}\left(z_{i}-\chi\left(z_{i}\right)\right)^{k}\left(U(\mathrm{~g}) \otimes_{U(\mathfrak{t})} V_{\gamma}\right) / \sum_{i=1}^{l}\left(z_{i}-\chi\left(z_{i}\right)\right)^{k+1}\left(U(\mathrm{~g}) \otimes_{U(\mathfrak{t})} V_{\gamma}\right)$.

Thus ker $\psi_{k+1}$ is isomorphic with a quotient of a direct sum of copies of $M_{\gamma, \chi, 1}$.

Since $M_{\gamma, \chi, k}$ is admissible and finitely generated, Theorem 5.7 implies that if $(\mathfrak{g}, \mathfrak{l})$ satisfies property $(\mathfrak{n})$ then $M_{\gamma, \chi, k}$ has a finite Jordan-Hölder series (a descending chain with irreducible quotients). Let $\Pi(\chi, \gamma)$ be the set of isomorphism classes of the constituents of the Jordan-Hölder series of $M_{\gamma, \chi, 1}$. The above observations imply

Lemma 5.9. Assume that $(\mathfrak{g}, \mathfrak{f})$ satisfies property $(\mathfrak{n})$. Then

(1) $|\Pi(\chi, \gamma)|<\infty$.

(2) The class of any subquotient of $M_{\gamma, \chi, k}$ is in $\Pi(\chi, \gamma)$.

We are now ready to prove the main result of this section.

TheOREM 5.10. Suppose that $(\mathfrak{g}, \mathfrak{f})$ satisfies property $(\mathfrak{n})$. Let $M$ be a $(\mathfrak{g}, \mathfrak{f})$-module such that $\operatorname{dim} M<\operatorname{card}(F)$. If $\mathfrak{n} M=M$ then $M=(0)$.

Proof. (1) If $V$ is a $\mathrm{g}$-module such that $\mathrm{n} V=V$ and if $\bar{V}$ is a quotient of $V$, then $\mathrm{n} \bar{V}=\bar{V}$.

This is clear. We display it since it will be used repeatedly in the proof of the result. Let $z_{1}, \ldots, z_{l}$ be the generators of $Z$ as above.

We will suppose that $\mathfrak{n} M=M$ but $M \neq 0$ and aim for a contradiction.

(2) There is a homomorphism $\chi: Z \rightarrow F$ so that $M$ has a quotient $\bar{M}$ on which $z-\chi(z)$ is locally nilpotent for all $z \in Z$.

Let $M^{\prime}$ be the $F\left[z_{1}\right]$-torsion submodule of $M$. If $M^{\prime} \neq M$ then $F\left[z_{1}\right]$ is torsion free on $M / M^{\prime}$. Hence Lemma 5.5 implies that there is a $\lambda_{1} \in F$ such that $z_{1}-\lambda_{1}$ is not surjective on $M / M^{\prime}$. Hence $z_{1}$ acts by $\lambda_{1}$ on $\left(M / M^{\prime}\right) /\left(z_{1}-\lambda_{1}\right)\left(M / M^{\prime}\right)$. We therefore see that $M$ has a quotient $\bar{M}$ so that $F\left[z_{1}\right]$ acts by pure torsion on $\bar{M}$. Arguing in the same way for $z_{1}, \ldots, z_{l}$ we see that $M$ has a nonzero quotient $\bar{M}$ such that if $\bar{m} \in \bar{M}$ then $\operatorname{dim} Z \cdot \bar{m}<\infty$. But then $\bar{M}=\oplus \bar{M}^{x}$. Fix $\chi$ so that $\bar{M}^{x}=0$. Then $\bar{M}^{x}$ is the desired quotient.

By (1) $n \bar{M}=\bar{M}$. Thus we may assume $\bar{M}=M$. Let $M^{\prime}$ be the finite dual of $M$. Let $\mu \in M^{\prime}, \mu \neq 0$, so that $U(\mathfrak{f}) \cdot \mu$ is irreducible as a $\mathfrak{f}^{*}$-module. Let $\gamma^{*}$ be the class of $U(\mathfrak{f}) \mu$. Set $\tilde{M}=U(\mathrm{~g}) \cdot \mu$. Let $N=\{v \in M \mid \tilde{M}(v)=0\}$. Then $\bar{M}=M / N$ still has the property that $\mathrm{n} \bar{M}=\bar{M}$ and $\bar{M} \neq(0)$. We may assume $\bar{M}=M$.

Set $M_{k}=\left\{m \in M \mid\left(z_{i}-\chi\left(z_{i}\right)\right)^{k} m=0, i=1, \ldots, l\right\}$. Then $M_{1} \subset M_{2} \subset \cdots$ and $\cup M_{i}=M$. Set $\tilde{M}_{k}=\left.\tilde{M}\right|_{M_{k}}=\left\{\left.\xi\right|_{M_{k}} \mid \xi \in \tilde{M}\right\}$. Set $\left({ }^{t} \chi\right)(z)=\chi\left({ }^{t} z\right)$. Then $\tilde{M}_{k}$ is a quotient of $M_{\gamma^{*}, \chi, k}$. Since $M_{\gamma^{*}, t^{\prime}, k}$ is admissible we see that

(3) $\tilde{M}_{k}$ is admissible.

Now $\tilde{M}_{k}=\tilde{M}_{k, 1} \supset \tilde{M}_{k, 2} \supset \cdots \supset \tilde{M}_{k, d} \supset \tilde{M}_{k, d+1}=(0)$ with $\tilde{M}_{k, j} / \tilde{M}_{k, j+1}$ irreducible and (the class of $\left.\tilde{M}_{k, j} / \tilde{M}_{k, j+1}\right) \in \Pi\left(\gamma^{*},{ }^{t} \chi\right)$. Set $\Pi\left(\gamma^{*},{ }^{t} \chi\right)^{*}$ equal to the set of equivalence classes of irreducible $(\mathfrak{g}, \mathfrak{f})$-modules whose $\mathfrak{l}$-finite duals are in $\Pi\left(\gamma^{*},{ }^{t} \chi\right)$. Then if $M_{k, j}=\left\{m \in M_{k} \mid \tilde{M}_{k, j}(m)=0\right\}$ we see that $M_{k}=M_{k, d+1} \supset$ $M_{k, d} \supset \cdots \supset M_{k, 1}=(0)$ and the class of $M_{k, j} / M_{k, j-1}$ is in $\Pi\left(\gamma^{*},{ }^{t} \chi\right)^{*}$. 
We therefore have

(4) $M_{k}$ is a finitely generated admissible $(\mathfrak{g}, \mathfrak{f})$-module and the equivalence class of every irreducible subquotient of $M_{k}$ is in the finite set $\Pi\left(\gamma^{*},{ }^{t} \chi\right)^{*}$.

(5) For each $k=1,2, \ldots$ there is $i(k)$ so that $M_{k} \subset \mathfrak{n} M_{i(k)}$.

Let $m_{1}, \ldots, m_{n}$ be generators of $M_{k}$ as a $U(\mathfrak{n})$-module. Then $m_{i} \in \mathfrak{n} M, i=1, \ldots, n$. Hence $m_{i} \in \mathfrak{n} M_{k_{i}}$ for some integers $k_{i}, i=1, \ldots, n$. Let $i(k)$ be the maximal of the $k_{i}$.

Then

$$
M_{k}=\sum U(\mathfrak{n}) m_{i} \subset \sum U(\mathfrak{n}) \mathfrak{n} M_{i(k)}=\mathfrak{n} M_{i(k)}
$$

Set

$$
j(k)=\underbrace{i(\ldots i(1))}_{k \text {-times }} .
$$

Then

(6) $M_{1} \subseteq \mathfrak{n}^{k} M_{j(k)}$.

We are finally ready to derive a contradiction. Put $E=\cup_{N \in \Pi\left(\gamma^{*}, t x\right)^{*}} E(N)$. Then $E$ is a finite set. Every weight $\mu$ of $M_{j(k)}^{*}[\mathfrak{n}]$ is of the form $\xi-Q, \xi \in E$ and $Q$ a sum of elements of $\Lambda^{+}$by 5.8. If $v \in M_{1}^{*}[\mathfrak{n}]$ then there is $v^{\prime} \in M_{j(k)}^{*}[\mathfrak{n}]$ so that $\left.v^{\prime}\right|_{M_{1}}=v$. Now $M_{1} \subset \mathfrak{n}^{k} M_{j(k)}$; hence $v^{\prime} \notin\left(M_{j(k)} / \mathfrak{n}^{k} M_{j(k)}\right)^{*}$. But then $\mu=\xi^{\prime}-\left(\lambda_{1}\right.$ $\left.+\cdots+\lambda_{r}\right)$, with $\xi^{\prime} \in E\left(M_{j(k)}\right), \lambda_{i} \in \Lambda^{+}$and $r \geqslant k$ by (1) in the proof of 5.6. Hence $\mu=\xi-\mu_{1}-\cdots-\mu_{s}, \xi \in E, \mu_{i} \in \Lambda^{+}$and $s \geqslant k$.

We therefore see that if $\mu$ is a weight of $j\left(M_{1}\right)$ then for each $k=1,2, \ldots$ there is $\xi_{k} \in E$ and $\mu_{k, 1}, \ldots, \mu_{k, s_{k}} \in \Lambda^{+}, s_{k} \geqslant k$, so that

$$
\mu=\xi_{k}-\sum_{i=1}^{s_{k}} \mu_{k, i} .
$$

Now argue as in the proof of (2) in 5.6 to obtain a contradiction.

Corollary 5.11. Suppose that $\left(\mathfrak{g} \otimes_{F} \tilde{F}, \mathfrak{f} \otimes_{F} \tilde{F}\right)$ has property $\left(\mathfrak{n} \otimes_{F} \tilde{F}\right)$ for all sufficiently large algebraically closed field extensions $\tilde{F}$ of $F$. If $M \neq 0$ is a $(\mathfrak{g}, \mathfrak{t})$ module then $\mathfrak{n} M \neq M$.

Proof. Let $\tilde{F}$ be an algebraically closed field extension of $F$ such that card $\tilde{F}>$ $\operatorname{dim}_{F} M$ and so large that $\left(\mathfrak{g} \otimes_{F} \tilde{F}, \mathfrak{g} \otimes_{F} \tilde{F}\right)$ has property $\left(\mathfrak{n} \otimes_{F} \tilde{F}\right)$. If $\tilde{M}=M \otimes_{F} \tilde{F}$, then Theorem 5.10 implies $\left(\mathfrak{n} \otimes_{F} \tilde{F}\right) \tilde{M}=\tilde{M}$. But then $\mathfrak{n} M \neq M$.

Note. It is not known to us whether $(\mathfrak{g}, \mathfrak{f})$ having property $(\mathfrak{n})$ for $F$ implies property $\left(\mathfrak{n} \otimes_{F} \tilde{F}\right)$ for $\left(g \otimes_{F} \tilde{F}, \mathfrak{f} \otimes_{F} \tilde{F}\right)$ when $\tilde{F}$ is an algebraically closed field extension of $F$. However, our proof of property $(n)$ for the cases covered in $\$ 6$ proves the result for every algebraically closed field.

6. Property $(\mathfrak{n})$ for $(\mathfrak{g} l(n, F), \mathfrak{g} o(n, F))$.In this section we give a proof of property $(\mathfrak{n})$ for $(\mathfrak{s} l(n, F), \mathfrak{s} o(n, F))$ for $F$ an algebraically closed field. Here $\mathfrak{n}_{n}$ is the Lie algebra of upper triangular $n \times n$ matrices over $F$ with zeros on the main diagonal. a is the space of diagonal matrices in $\mathfrak{g} l(n, F)$. We prove this result simultaneously with Theorem 4 of the introduction. The proof rests on several lemmas. 
If $V$ is a vector space over $F$ we say that $T \in \operatorname{End}(V)$ is nilpotent if for each $v \in V$ there is a positive integer $k$ (depending on $v$ ), such that $T^{k} v=0$. If $R$ is an associative algebra over $F$ then $T \in R$ is said to be nilpotent if ad $T: R \rightarrow R$ is nilpotent.

LEMMA 6.1. Let $R$ be an associative algebra over $F$. Let $x \in R$ be nilpotent. If $M$ is an $R$-module then $M[x]=\left\{m \in M \mid x^{k} m=0\right.$ for some $\left.k\right\}$ is an $R$-submodule of $M$.

Proof. If $m \in M[x], r \in R$, then $x^{k} m=0,(\operatorname{ad} x)^{l} \cdot r=0$ for some positive integers $k$ and $l$. Hence $x^{k+l}(r m)=0$.

LEMMA 6.2. Let $R$ and $x$ be as in Lemma 6.1. Suppose that $M$ is an $R$-module such that for each $R$-module quotient $\bar{M}$ of $M$ there is $0 \neq m \in \bar{M}$ such that $x \bar{m}=0$. Then $x$ acts nilpotently on $M$.

Proof. Let $M[x]$ be as in 6.1 . Then $x$ clearly acts injectively on $M / M[x]$. Hence $M / M[x]=(0)$ by the hypothesis.

Lemma 6.3. Let $V$ be a vector space over $F$. Let $X, Y, C \in \operatorname{End}(V)$. Suppose that

(1) $[C, X]=Y,[C, Y]=-X$ and $[X, Y]=0$.

(2) $C$ acts semisimply on $V$.

(3) There exists $v \neq 0, v \in X V$, with $C V=\lambda v$ for some $\lambda \in F$.

Then $X^{2}+Y^{2}$ is not injective on $V$.

Proof. Set $e^{+}=\frac{1}{2}(X-i Y), e^{-}=\frac{1}{2}(X+i Y)$. Then $\left[C, e^{ \pm}\right]= \pm i e^{ \pm}$. By assumption $v=X w$ for some $w \in V$. Now $w=\sum w_{\mu}$ with $C w_{\mu}=\mu w_{\mu}$. Hence $v=\sum e^{+} w_{\mu}+$ $\sum e^{-} w_{\mu}$. Now $w=\sum_{r=k}^{l} w_{\lambda+i r}+\sum_{\mu \notin \lambda+i Z} w_{\mu}$, with $w_{\lambda+i l} \neq 0$ and $w_{\lambda+i k} \neq 0$. Clearly, $v=\sum_{r=k}^{l} e^{+} w_{\lambda+i r}+\sum_{r=k}^{l} e^{-} w_{\lambda+i r}$. If $l \geqslant 0$ then it is clear that $e^{+} w_{\lambda+i l}=0$. If $l<0$ then $e^{-} w_{\lambda+i k}=0$. In any event $e^{+} e^{-}=\frac{1}{4}\left(X^{2}+Y^{2}\right)$ is not injective.

Corollary 6.4. Let $X, Y, C, V$ be as in Lemma 6.3. If $X V=V$ then $X^{2}+Y^{2}$ acts nilpotently on $V$.

Proof. Let $R$ be the subalgebra of $\operatorname{End}(V)$ generated by $X, Y$ and $C$. Clearly, $X^{2}+Y^{2}$ is a nilpotent element of $R$. If $\bar{V}$ is an $R$-module quotient of $V$ then $X \bar{V}=\bar{V}$. Hence Lemma 6.3 implies that $X^{2}+Y^{2}$ has a nonzero kernel on $\bar{V}$. But then Lemma 6.2 implies that $X^{2}+Y^{2}$ is nilpotent on $V$.

Let $\mathfrak{g}_{n}$ denote the semidirect product $\mathfrak{g} o(n, F) \oplus F^{n}\left(F^{n}\right.$ abelian) with $[X, v]=X v$ for $X \in \mathfrak{s} o(n, F), v \in F^{n}$. Let $e_{1}, \ldots, e_{n}$ denote the standard basis of $F^{n}$.

LeMma 6.5. Let $M$ be $a\left(\mathfrak{g}_{n}, \mathfrak{s o}(n, F)\right)$-module. Assume that $n \geqslant 2$ and that $M=\sum_{i=1}^{n-1} e_{i} M$. Then there exists an element $f \in U\left(F^{n}\right)$ of the form $f=e_{n}^{2 k}+$ $\sum_{j=0}^{k-1} e_{n}^{2 j} g_{j}\left(e_{1}, \ldots, e_{n-1}\right)$ that acts nilpotently on $M$.

Proof. Put $M_{0}=(0), M_{r}=\sum_{j=1}^{r} e_{j} M$. Set $\bar{M}_{r}=M / M_{r}$. Then $\bar{M}_{0}=M$. We assume that $M_{r}=M, M_{r-1} \neq M$. By assumption $r \leqslant n-1$. Clearly $e_{r} \bar{M}_{r-1}=\bar{M}_{r-1}$. Let, for $1 \leqslant i<j \leqslant n, c_{i j} \in \mathfrak{g} o(n, F)$ be defined by $c_{i j} e_{j}=e_{j}, c_{i j} e_{j}=-e_{i}$ and $c_{i j} e_{k}=0, k \neq i, j$. Then $c_{i j}$ acts semisimply on $M$. Clearly $c_{r, n} M_{r-1} \subset M_{r-1}$. Corollary 6.4 implies that $e_{r}^{2}+e_{n}^{2}$ acts nilpotently on $\bar{M}_{r-1}$. 
Suppose that we have shown that

$$
\left(e_{r-k}^{2}+\cdots+e_{r}^{2}+e_{n}^{2}\right)\left(e_{r-k}^{2}+\cdots+e_{r-1}^{2}+e_{n}^{2}\right) \cdots\left(e_{r-k}^{2}+e_{n}^{2}\right)
$$

acts nilpotently on $\bar{M}_{r-k-1}$ and $r-k-1 \geqslant 1$. Then $e_{r-k-1} \bar{M}_{r-k-1}=0$ implies that

$$
\begin{aligned}
h= & \left(e_{r-k-1}^{2}+e_{r-k}^{2}+\cdots+e_{r}^{2}+e_{n}^{2}\right)\left(e_{r-k-1}^{2}+\cdots+e_{r-1}^{2}+e_{n}^{2}\right) \\
& \cdots\left(e_{r-k-1}^{2}+e_{r-k}^{2}+e_{n}^{2}\right)
\end{aligned}
$$

acts nilpotently on $\bar{M}_{r-k-1}$.

Let $(0) \neq N$ be a $U\left(F c_{r-k-1, n} \otimes F^{n}\right)$-quotient module of $\bar{M}_{r-k-2}$. We note that $\left[c_{r-k-1, n}, h\right]=0$. If $w \in N$ there is $l$ so that $h^{l} w \in e_{r-k-1} N$. Thus there is $v \neq 0$ so that

$$
c_{r-k-1, n} v=\lambda v \text { and } h \cdot v=e_{r-k-1} v^{\prime}
$$

for some $v^{\prime} \in N$. Either $h \cdot v=0$ or Lemma 6.3 implies that $e_{r-k-1}^{2}+e_{n}^{2}$ has a nonzero kernel on $N$. Hence $h\left(e_{r-k-1}^{2}+e_{n}^{2}\right)$ is not injective on $N$. Lemma 6.2 now implies that $h\left(e_{r-k-1}^{2}+e_{n}^{2}\right)$ is nilpotent on $\bar{M}_{r-k-2}$.

We therefore have by induction that

$$
f=\left(e_{1}^{2}+\cdots+e_{r}^{2}+e_{n}^{2}\right)\left(e_{1}^{2}+\cdots+e_{r-1}^{2}+e_{n}^{2}\right) \cdots\left(e_{1}^{2}+e_{n}^{2}\right)
$$

is nilpotent on $\bar{M}_{0}=M$. Clearly $f$ is of the desired form.

Let $\mathfrak{p}_{n}$ be the semidirect product of $g l(n, F)$ with the abelian Lie algebra $F^{n}$ with $[X, v]=X v, X \in g l(n, F), v \in F^{n}$. Let $\mathrm{n}_{n}$ be the Lie algebra of upper triangular matrices in $g l(n, F)$ with zeros along the main diagonal.

We look at $\mathfrak{p}_{n}$ as a Lie subalgebra of $g l(n+1, F)$ as follows: If $v \in F^{n}$ then we look upon $v$ as an $n \times 1$ column vector. If $(X, v) \in \mathfrak{p}_{n}$ then we identify it with

$$
\left[\begin{array}{ll}
X & v \\
0 & 0
\end{array}\right] \in g l(n+1, F) \text {. }
$$

Then $\mathfrak{n}_{n+1}=\mathfrak{n}_{n} \oplus F^{n}$ with this identification.

TheOREM 6.6. Assume that $F$ is uncountable. If $(0) \neq M$ is $a(g l(n, F), \mathfrak{s} o(n, F))$ module finitely generated over $U\left(\mathfrak{n}_{n}\right)$ then $\mathfrak{n} M \neq M$.

THEOREM 6.7. Assume that $F$ is uncountable. If $(0) \neq M$ is $a\left(\mathfrak{p}_{n}, \lessgtr o(n, F)\right)$-module finitely generated under $U\left(n_{n} \oplus F^{n}\right)$ then $\left(n_{n} \oplus F^{n}\right) M \neq M$.

We prove Theorems 6.6 and 6.7 simultaneously by induction on $n$. We first note

(1) If Theorem 6.6 is true for $n$, then $(\mathfrak{g} l(n, F), \mathfrak{g} o(n, F))$ has property $\left(\mathfrak{n}_{n}\right)$.

Indeed, $g l(n, F)=F I \oplus \mathfrak{g} l(n, F)$. Let $(0) \neq M$ be a finitely generated, admissible, $(\xi l(n, F), \mathfrak{s} o(n, F))$-module. Define $I M=0$. Then $M$ is a $(g l(n, F), \mathfrak{s} o(n, F))$-module finitely generated over $U\left(\mathfrak{n}_{n}\right)$ (see Theorem 5.2). Hence $\mathrm{n}_{n} M \neq M$.

(2) If Theorem 6.7 is true for $n \leqslant k$ then Theorem 6.6 is true for $n \leqslant k+1$.

Indeed, a $(g l(n+1, F), \mathfrak{s} o(n+1, F))$-module $M$, finitely generated under $U\left(\mathfrak{n}_{n+1}\right)$, is clearly a $\left(\mathfrak{p}_{n}, \mathfrak{s} o(n, F)\right)$-module finitely generated under $U\left(\mathfrak{n}_{n} \oplus F^{n}\right)$. 
We now proceed with the proof of Theorems 6.6 and 6.7. If $n=1$ then Theorem 6.7 follows from Proposition 3.1. We assume Theorem 6.7 for $1 \leqslant n \leqslant k$ and consider the case $n=k+1$. Let $M \neq(0)$ be a $\left(\mathfrak{p}_{n}, \mathfrak{s} o(n, F)\right)$-module finitely generated over $U\left(\mathfrak{n}_{n} \oplus F^{n}\right)$ such that $\left(\mathfrak{n}_{n} \oplus F^{n}\right) M=M$.

(3) $F^{n} M=M$.

Indeed, $\bar{M}=M / F^{n} M$ is a $(g l(n, F), \mathfrak{s} o(n, F))$-module finitely generated under $U\left(\mathfrak{n}_{n}\right)$ such that $\mathfrak{n}_{n} \bar{M}=\bar{M}$. Hence the inductive hypothesis and (2) imply that $\bar{M}=(0)$.

Suppose now that $M=\sum_{i=1}^{n-1} e_{i} M$. Lemma 6.5 says that there is $f=e_{n}^{2 k}+$ $\sum_{j=0}^{k-1} e_{n}^{2 j} g_{j}\left(e_{1}, \ldots, e_{n-1}\right)$ acting nilpotently on $M$.

The inductive hypothesis combined with (1), (2) imply that $(\mathfrak{I l}(n, F), \mathfrak{g} o(n, F))$ has property $\left(\mathfrak{n}_{n}\right)$. We partition the elements $x \in \mathfrak{s} l(n, F)$ in the following way:

$$
x=\left[\begin{array}{ll}
A & b \\
d & c
\end{array}\right]
$$

with $A \in \mathfrak{g} l(n-1, F)$. Set $q=\left\{\left[\begin{array}{ll}0 & b \\ 0 & 0\end{array}\right] \mid\right.$ with $b$ an $n-1 \times 1$ matrix $\}$. Theorem 5.10 implies that $q M \neq M$. Since $f$ above acts nilpotently on $M, M$ is finitely generated as a $U\left(n_{n} \oplus \sum_{i=1}^{n-1} F e_{i}\right)$-module.

We identify $g l(n-1, F)$ with the space of all

$$
X=\left\{\left[\begin{array}{ll}
A & 0 \\
0 & 0
\end{array}\right] \mid A \in \mathfrak{g} l(n-1, F)\right\} \subset \mathfrak{g} l(n, F) .
$$

Then $\bar{M}=M / \mathfrak{q} M$ is a $\left(\mathfrak{g} l(n-1, F) \oplus \sum_{i=1}^{n-1} F e_{i}, \mathfrak{g} o(n-1, F)\right)$-module finitely generated under $U\left(\mathfrak{n}_{n-1} \oplus \sum_{i=1}^{n-1} F e_{i}\right)$ and $\left(\mathfrak{n}_{n-1} \oplus \sum_{i=1}^{n-1} F e_{i}\right) \bar{M}=\bar{M}$. The inductive hypothesis now implies the contradiction that $\bar{M}=(0)$. We have therefore shown

(4) $\sum_{i=1}^{n-1} e_{i} M \neq M$.

We identify $\mathfrak{p}_{n-1}$ with the corresponding subalgebra of $g l(n, F)$ as before the statement of Theorem 6.6.

Let $h$ be the element of $g l(n, F)$ with exactly one nonzero entry which is in the $n$, $n$ position. Then $\left[\mathfrak{p}_{n-1}, \sum_{i=1}^{n-1} F e_{i}\right] \subset \sum_{i=1}^{n-1} F e_{i}$. Let

$$
\bar{e}_{n}=e_{n}+\sum_{i=1}^{n-1} F e_{i} \in F^{n} / \sum_{i=1}^{n-1} F e_{i} .
$$

Then $\bar{M}=M / \sum_{i=1}^{n-1} e_{i} M$ is a $\left(\mathfrak{p}_{n-1} \oplus F \bar{e}_{n} \oplus F h, \mathfrak{g} o(n-1, F)\right)$-module.

We note that $n_{n} \subset \mathfrak{p}_{n-1}$ as usual and $\bar{M}$ is finitely generated as a $U\left(\mathfrak{n}_{n} \oplus F \bar{e}_{n}\right)$ module.

(5) $\mathfrak{n}_{n} \bar{M}=\bar{M}$.

Indeed, set $N=\bar{M} / \mathfrak{n}_{n} \bar{M}$. Then $N$ is a $\left(F h \oplus F \bar{e}_{n}\right)$-module finitely generated as a $U\left(F \bar{e}_{n}\right)$-module and $\bar{e}_{n} N=N$. Proposition 3.1 implies that $N=(0)$.

Let $V$ be a nonzero irreducible quotient of $\bar{M}$ as a $\left(\mathfrak{p}_{n-1} \oplus F \bar{e}_{n}\right)$-module. Now $\left[\mathfrak{p}_{n-1}, \bar{e}_{n}\right]=0$. Hence Lemma 5.5 implies that $\bar{e}_{n}$ acts by a scalar $\lambda$ on $V$. Hence $V$ is a $\left(\mathfrak{p}_{n-1}, \mathfrak{S} o(n-1, F)\right)$-module finitely generated under $U\left(\mathfrak{n}_{n-1} \oplus F^{n-1}\right)$ and $\left(n_{n-1} \oplus F^{n-1}\right) V=V$. This implies $V=(0)$. A contradiction. This contradiction completes the inductive step and hence the proof of Theorems 6.6 and 6.7.

If $M$ is an irreducible finite dimensional $\mathfrak{p}_{n}$-module then it is well known that $F^{n} \cdot M=(0)$. Our first corollary is a generalization of this fact. 
COROLlary 6.8. Let $F$ be algebraically closed and uncountable. If $M$ is an irreducible $\left(\mathfrak{p}_{n}, \mathfrak{s} o(n, F)\right)$-module finitely generated as a $U\left(\mathfrak{n}_{n} \oplus F^{n}\right)$-module, then $F^{n} M=(0)$.

Proof. $F^{n} M$ is a $\left(\mathfrak{p}_{n}, \mathfrak{s o}(n, F)\right)$-submodule of $M$. Theorem 6.7 implies that $F^{n} M \neq M$. Hence $F^{n} M=(0)$.

COROLlaRY 6.9. Let $F$ be algebraically closed (but not necessarily uncountable). If $(0) \neq M$ is $a\left(\mathfrak{p}_{n}, \mathfrak{s} o(n, F)\right)$-module finitely generated over $U\left(\mathfrak{n}_{n} \oplus F^{n}\right)$ then

$$
\left(n_{n} \oplus F^{n}\right) M \neq M \text {. }
$$

Proof. Let $\tilde{F}$ be an uncountable algebraically closed extension of $F$. Suppose $M$ is as in the statement but that $\left(\mathfrak{n}_{n} \oplus F^{n}\right) M=M$. Then $M \otimes_{F} \tilde{F}$ is a $\left(\mathfrak{p}_{n} \otimes_{F} \tilde{F}\right.$, $\vdots o(n, \tilde{F}))$-module finitely generated under $U\left(\mathrm{n}_{n} \otimes_{F} \tilde{F} \oplus \tilde{F}^{n}\right)$ and

$$
\left(\mathrm{n}_{n} \otimes_{F} \tilde{F} \oplus \tilde{F}^{n}\right) M=M \text {. }
$$

Hence Theorem 6.7 implies a contradiction.

COROllary 6.10. Let $F$ be an algebraically closed field of characteristic 0 . Then $(\mathfrak{g} l(n, F), \mathfrak{g} o(n, F))$ satisfies property $\left(\mathfrak{n}_{n}\right)$.

PROOF. In light of Corollary 6.9 the arguments proving (1) and (2) of the proof of 6.7 and 6.8 prove the corollary.

Notice that the only property of admissible finitely generated $(\mathfrak{g}, \mathfrak{f})$-modules that we have used in this section is that they are finitely generated as $U(\mathfrak{n})$-modules. We end this section by showing that the two properties are equivalent.

THEOREM 6.11. Suppose that $(\mathfrak{g}, \mathfrak{f})$ satisfies property $(\mathfrak{n})$ and that $M$ is a finitely generated $(g, \mathfrak{f})$-module. Then the following are equivalent:

(1) $M$ is admissible.

(2) $M$ is finitely generated as a $U(\mathfrak{n})$-module.

Proof. We need only show that (2) implies (1) (see Theorem 5.2). Suppose $M$ satisfies (2). Then $N=\cap_{k=1}^{\infty} \mathfrak{n}^{k} M$ is a $g$-submodule of $M$ and thus $N$ is a finitely generated $U(\mathrm{~g})$-module. Thus if $N \neq(0)$ then $N$ has an irreducible quotient $\bar{N}$. Property (n) implies that $\mathfrak{n} \bar{N} \neq \bar{N}$. Hence $\mathfrak{n} N \neq N$. On the other hand Proposition 2.1 implies that there is a positive integer $k$ so that $\mathfrak{n}^{k} M \cap N \subset \mathfrak{n} N$. But $N \subset \mathfrak{n}^{k} M$. Hence $\mathrm{n} N=N$, a contradiction. We have thus shown that $N=(0)$. Theorem 5.6(ii) now implies that if $m \in M$ then $\operatorname{dim} Z \cdot m<\infty$. Theorem 5.3 now applies and says that $M$ is admissible.

\section{REFERENCES}

1. M. F. Atiyah and I. G. Macdonald, Introduction to commutative algebra, Addison-Wesley, Reading, Mass., 1969.

2. W. Casselman and M. S. Osborne, The restriction of admissible representations to n, Math. Ann. 233 (1978), 193-198.

3. J. Dixmier, Enveloping algebras, North-Holand, Amsterdam, 1977.

4. B. Kostant, On Whittaker vectors and representation theory, Invent. Math. 48 (1968), 101-184.

5. J. Lepowsky, Algebraic results on representions of semi-simple Lie groups, Trans. Amer. Math. Soc. 176 (1973), 1-44. 
6. Conical vectors in induced modules, Trans. Amer. Math. Soc. 208 (1975), 219-272.

7. J. C. McConnell, Localization in enveloping rings, J. London Math. Soc. 43 (1968), 421-428; (2) 3 (1971), 409-410.

8. D. Miličć, Notes on asymptotics of admissible representations of semi-simple Lie groups, Institute for Advanced Study, Princeton, N.J., 1976.

9. G. Warner, Harmonic analysis on semisimple Lie groups. I, Die Grundlehren Math. Wissenschaften, Band 188, Springer-Verlag, Berlin, 1972.

10. O. Zariski and P. Samuel, Commutative algebra. I, Van Nostrand, Princeton, N.J., 1958.

Department of Mathematics, Gonville and Caius College, Cambridge CB2 1TA, England

Department of Mathematics, Rutgers University, New Brunswick, NeW Jersey 08903 